\title{
A preoperative evaluation for neo-infantile liver tumors using a three-dimensional reconstruction of multidetector row $\mathrm{CT}$
}

\author{
YOSHIAKI KINOSHITA ${ }^{1}$, RYOTA SOUZAKI ${ }^{1}$, TATSURO TAJIRI ${ }^{1}$, SATOSHI IEIRI $^{1,2}$, \\ MAKOTO HASHIZUME ${ }^{2}$ and TOMOAKI TAGUCHI ${ }^{1}$ \\ ${ }^{1}$ Department of Pediatric Surgery, Graduate School of Medical Sciences, Kyushu University, ${ }^{2}$ Center for the Integration \\ of Advanced Medicine and Innovative Technology, Kyushu University Hospital, Fukuoka 812-8582, Japan
}

Received September 5, 2008; Accepted December 9, 2008

DOI: $10.3892 /$ or_00000298

\begin{abstract}
Multidetector row CT (MDCT), which has been used extensively in adult patients, has also recently been used for the evaluation of children. As pediatric surgeons, we preoperatively examined 10 cases of liver tumors by MDCT and performed three-dimensional reconstruction and a volumetric analysis. Instead of angiography, which requires general anesthesia in children, this method can provide a fine image of the anatomy between the tumor and the vessels, as well as identify the presence of any anomalous vascular branches. It also makes it possible to calculate the residual liver volume for the proposed operation and to determine the optimal cut line. However, there are still certain problems associated with pediatric cases, including the determination of the appropriate volume of contrast medium, the occurrence of allergic reactions to the contrast medium, and the timing of enhancement. The resolution of the specific problems in the pediatric application of MDCT, and the development of a more effective procedure is thus required.
\end{abstract}

\section{Introduction}

Currently multidetector row CT (MDCT) has made it possible to obtain more valuable information than was previously possible with conventional CT. The MDCT is superior in spatial and temporal resolutions. In adult cases, the MDCT has been frequently applied as a diagnostic tool for tumors. In pediatric cases, however, some such problems as the necessity to use anesthesia, difficulty of respiratory restriction, determining the adequate volume of contrast medium, and the estimated irradiation have been recognized, the application of

Correspondence to: Dr Yoshiaki Kinoshita, Department of Pediatric Surgery, Graduate School of Medical Sciences, Kyushu University, 3-1-1, Maidashi, Higashi-ku, Fukuoka 812-8582, Japan E-mail: kinoppy@pedsurg.med.kyushu-u.ac.jp

Key words: multidetector row CT, liver tumor, angiography, volumetry, child
MDCT procedures in the pediatric population has therefore been limited. The development of the multidetector (16 or 64 slice spirals) has solved some of these problems. This study presents 10 cases of pediatric liver tumors, which were evaluated by MDCT using three-dimensional reconstruction and volumetry.

\section{Patients and methods}

In our department, 10 cases of neo-infantile liver tumors were admitted from 2003 to 2007 . Each case was examined preoperatively by MDCT. The clinical data of the patients are summarized in Table I. Regarding the age, 1 case was a neonate, 6 cases were infants younger than 1 year, and 3 cases were infants older than 1 year. Histologically, 7 cases had hepatoblastomas, 2 cases had infantile hemangioendotheliomas, and the remaining case was a malignant rhabdoid tumor. Six cases were treated by preoperative chemotherapy with cisplatin (CDDP) and pirarubicin (THP-adriamycin). Preoperative angiography with a transcatheter arterial chemoembolization (TACE) was performed for 5 cases, which showed insufficiency only for the preoperative systemic chemotherapy.

The scanning was performed by an MDCT with 64 detectors. As the contrast medium, iopamidole $(300 \mathrm{mg} / \mathrm{ml})$ was administered intravenously for $20 \mathrm{sec}$ with a dose of 2 $\mathrm{ml} / \mathrm{kg}$. The condition of collimation was 1 or $2 \mathrm{~mm}$ and the herical pitch was 5.5. The arterial phase, equilibrium phase, and portal venous phase were captured in that order.

Triclofossodium syrup or pentobarbital suppositories were routinely used as sedative agents. Intravenous ketamine instillation was added in some cases. All cases were well sedated and high quality images were acquired under spontaneous respiration.

The images from MDCT were transferred to a 3D workstation (Virtual Place Advance, AZE) and then were analyzed. By this method, the feeding vessels and accessory or aberrant vessels were evaluated. In addition, the vessel architecture, including the arterial system and portal venous system were independently demonstrated and reconstructed as each phase analysis. As a volumetric 3D-rendered analysis, the standard liver volume and residual liver volume for several patterns of proposed operation were calculated (1). The standard liver volume (SLV) was also calculated by the Urata equation: 
Table I. Clinical data of each case.

\begin{tabular}{|c|c|c|c|c|c|c|}
\hline Case & Age & Gender & Histology & Location of tumor & Preoperative chemotherapy & Angio + TACE \\
\hline 1 & 14 days & $\mathrm{F}$ & HB & Rt. lobe & ND & ND \\
\hline 2 & 3 months & $\mathrm{F}$ & $\mathrm{HB}$ & $\mathrm{S} 3,4,5,6$ & Done & Done \\
\hline 3 & 4 months & M & HB & Lt. lobe & Done & Done \\
\hline 4 & 8 months & $\mathrm{F}$ & HB & Lt. lobe & Done & Done \\
\hline 5 & 1 year & $\mathrm{F}$ & HB & Rt. lobe & Done & ND \\
\hline 6 & 1 year & M & HB & Rt. lobe & ND & ND \\
\hline 7 & 3 years & M & HB & Rt. lobe & Done & Done \\
\hline 8 & 5 months & M & MRT & Rt. lobe, $\mathrm{S} 4$ & Done & Done \\
\hline 9 & 1 month & M & HET & $\mathrm{S} 3$ & ND & ND \\
\hline 10 & 1 month & $\mathrm{F}$ & HET & Bil. lobe & ND & ND \\
\hline
\end{tabular}

HB, hepatoblastoma; MRT, malignant rhabdoid tumor; HET, hemangioendothelioma; ND, not done; TACE, transcatheter arterial chemoembolization.

Table II. Abnormal vessels demonstration and volumetry analysis for each case.

\begin{tabular}{|c|c|c|c|c|c|}
\hline Case & $\begin{array}{c}\text { Abnormal vessels } \\
\text { demonstration by MDCT }\end{array}$ & Proposed operation & $\operatorname{SLV}(\mathrm{ml})$ & RLV (ml) & $\operatorname{RLV}(\%)$ \\
\hline 1 & None & Rt. lobectomy & 114.1 & 67.4 & 59.0 \\
\hline 2 & $\mathrm{SMA} \rightarrow \mathrm{RHA}, \mathrm{LGA} \rightarrow \mathrm{LHA}$ & Central bisegmentectomy & 497.2 & 294.5 & 59.2 \\
\hline 3 & None & Lt. lobectomy & 267.9 & 213.1 & 79.0 \\
\hline 4 & None & Extended lt.lobectomy & 296.5 & 160.0 & 54.0 \\
\hline 5 & Lt. IVC & Rt. lobectomy & 284.1 & 190.4 & 67.0 \\
\hline 6 & None & Rt. lobectomy & 109.2 & 68.1 & 62.4 \\
\hline 7 & None & Rt. lobectomy & 398.6 & 259.1 & 65.0 \\
\hline 8 & $\mathrm{CA} \rightarrow \mathrm{MHA}, \mathrm{LGA} \rightarrow \mathrm{LHA}$ & Extended rt. lobectomy & 233.0 & 95.3 & 40.9 \\
\hline 9 & None & Tumor extirpation & 176.8 & 161.3 & 91.2 \\
\hline 10 & None & Biopsy & NA & NA & NA \\
\hline
\end{tabular}

SMA, superior mesenteric artery; RHA, right hepatic artery; LGA, left gastric artery; LHA, left hepatic artery; CA, celiac artery; MHA, middle hepatic artery; SLV, standard liver volume; RLV, residual liver volume; NA, not analyzed.

Table III. Advantages for each case obtained by MDCT reconstruction.

\begin{tabular}{|c|c|c|c|c|}
\hline Case & $\begin{array}{c}\text { Detection of abnormal } \\
\text { vessels or feeding arteries }\end{array}$ & $\begin{array}{l}\text { No need for } \\
\text { angiography }\end{array}$ & $\begin{array}{l}\text { Advantage by simultaneous } \\
\text { visualization of each phase }\end{array}$ & $\begin{array}{c}\text { Advantage by simulation of } \\
\text { proposed operation }\end{array}$ \\
\hline 1 & & 0 & 0 & \\
\hline 2 & 0 & & 0 & 0 \\
\hline 3 & & & 0 & \\
\hline 4 & & & 0 & 0 \\
\hline 5 & 0 & 0 & 0 & \\
\hline 6 & & 0 & 0 & \\
\hline 7 & & & 0 & \\
\hline 8 & 0 & & 0 & 0 \\
\hline 9 & 0 & 0 & 0 & \\
\hline 10 & 0 & 0 & 0 & \\
\hline
\end{tabular}


a

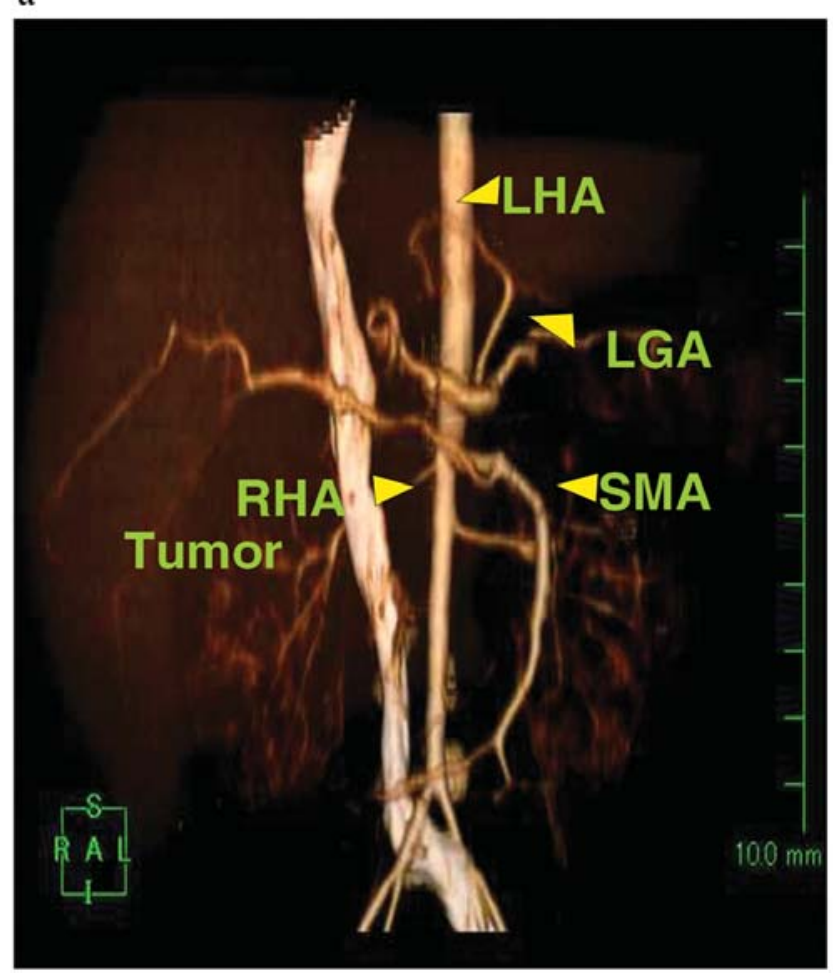

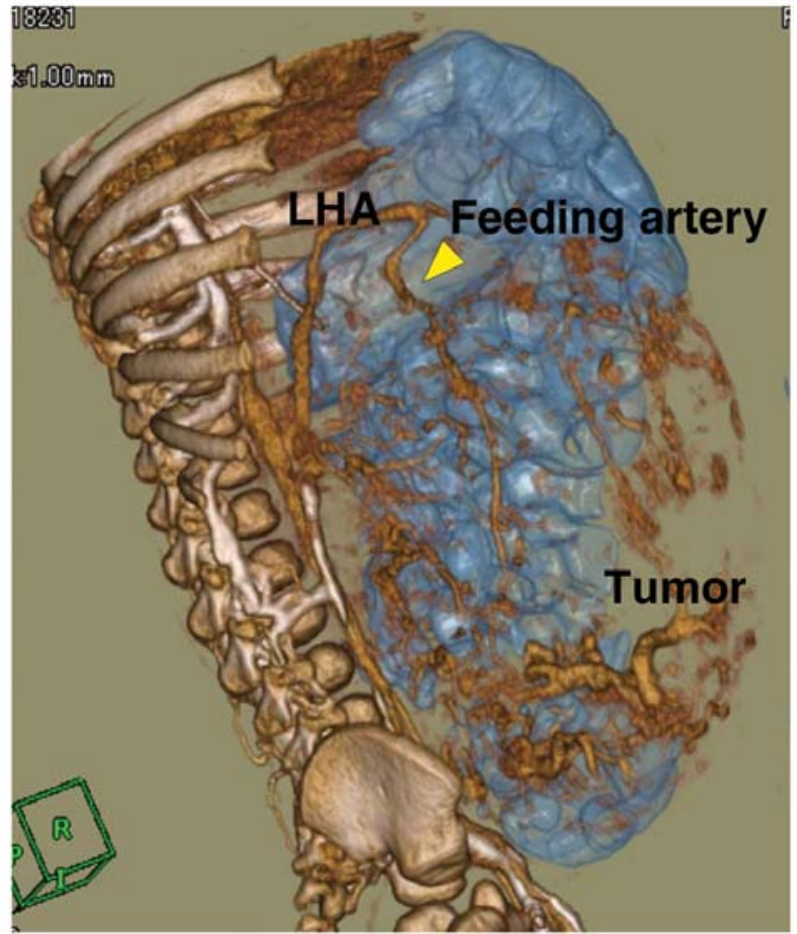

Figure 1. Detection of anomalous vessels and feeding vessels. (a) Case 6 (hepatoblastoma). The left hepatic artery (LHA) was observed to branch from the left gastric artery (LGA). The right hepatic artery (RHA) branched from the superior mesenteric artery (SMA). (b) Case 9 (hemangioendothelioma). The tumor feeding artery from the left hepatic artery (LHA) is detected by 3-D reconstruction images.

$\operatorname{SLV}(\mathrm{ml})=706.2 \mathrm{x}$ body surface area $\left(\mathrm{m}^{2}\right)(2)$. These reconstruction and volumetric analyses were performed by ourselves, namely by the 'pediatric surgeons'.

\section{Results}

Detection of anomalous vessels and feeding vessels. Abnormal vessels were detected in 3 cases (cases 2, 5, 8) (Table II). In case 2 , the reconstructed image detected anomalous branching in the left hepatic artery (LHA) arising from left gastric artery (LGA) and the right hepatic artery (RHA) arising from the superior mesenteric artery (SMA) (Fig. 1a). In the same way, anomalous branching vessels were detected in cases 5 and 8 . Furthermore, this procedure also clearly demonstrated the feeding vessels of huge tumors in cases 9 and 10 . In case 9 , the huge tumor was fed by a branching artery from the LHA (Fig. 1b). Those images were never inferior to those obtained from angiography and were very informative for the operation. In this manner, abnormal vessels or feeding arteries were detected in 5 cases. Since this vascular informations obtained by the reconstruction of the MDCT images was sufficient, conventional angiography was unnecessary for these 5 cases (Table III).

Each phase analysis. As shown by the images of case 5 (a oneyear-old girl with a hepatoblastoma in the right lobe) (Fig. 2a), each contrast phase, arterial phase (Fig. 2b), equilibrium phase (Fig. 2c), and portal venous phase (Fig. 2d) were independently extracted or combined as three-dimensional images, respectively (Fig. 2e). By this method, the precise anatomical association between the tumor and vessels could be clearly demonstrated simultaneously. Since the simultaneous evaluation of identical images is impossible by conventional $\mathrm{CT}$, this advantage was thus considered to be remarkable in all cases (Table III).

Volumetry analysis. The residual liver volume was calculated by a volumetric analysis for all cases (Table II). Since the whole liver was occupied by the tumor in case 10 , the proposed operation was a biopsy. In the other 6 cases (cases $1,3,5,6,7,9)$, the residual liver volume was calculated as $>50 \%$ in the initially proposed operative procedure. Therefore, an optimal cut line was defined by this volumetric analysis. However, in the remaining cases (cases 2, 4, 8), the cut lines were variously simulated in several ways, because a sufficient residual liver volume was not expected by means of an extended operation. For example, in case 2, the tumor occupied the central area of the liver and it was attached to the portal vein (Fig. 3a); therefore, various operative procedures were simulated. The standard liver volume of the patient was calculated at $497.2 \mathrm{ml}$. If the patients would have undergone a left trisegmentectomy, then the residual liver volume would only have been $28.3 \%$ of the standard liver volume (Fig. 3b). On the other hand, if a central bisegmentectomy could be performed, then the residual liver volume was estimated to be $59.2 \%$ of the standard liver volume (Fig. 3c). Therefore, 
a

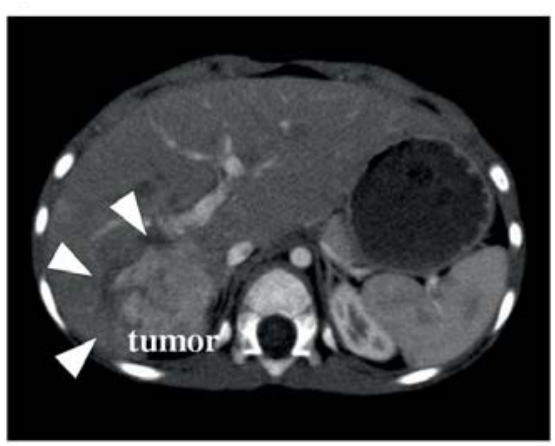

d

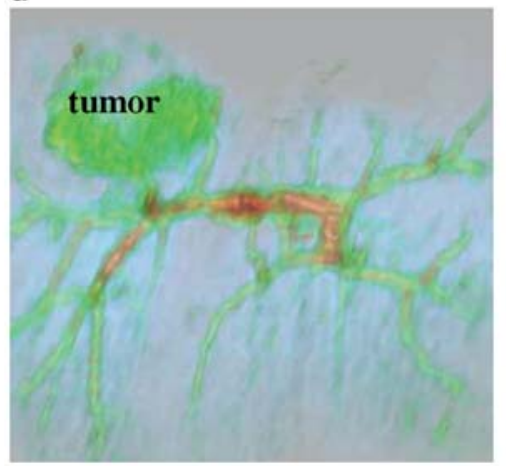

b

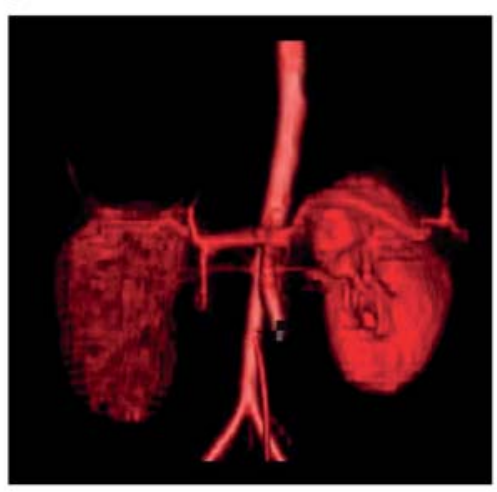

c

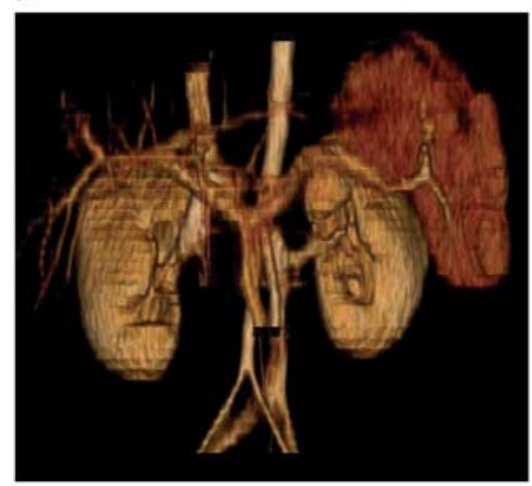

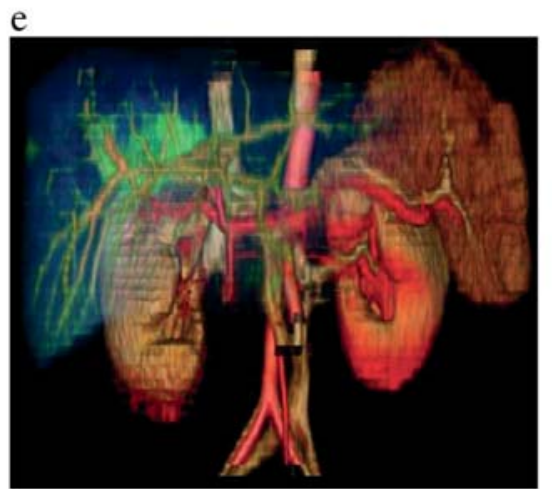

Figure 2. Each phase analysis (Case 5). (a) A 2-D image from a conventional CT. (b) A reconstructed image of the arterial phase. (c) A reconstructed image of the equilibrium phase. (d) A reconstructed image of the portal venous phase. (e) A reconstructed image of the combined multiphase images.

the central bisegmentectomy was selected for the preservation of postoperative liver function. Using these analyses, the most appropriate operation was performed in all cases.

\section{Discussion}

MDCT has multiple detectors along the body axis and simultaneouly provides a vast amount of information during one rotation of the X-ray tube. In comparison to the conventional helical CT, MDCT is superior in both temporal and spatial resolution (3). The reconstruction of images provides much more information without the degradation of images, and the amount of radiation exposure can also be reduced (4).

The usefulness of MDCT for the evaluation of pediatric disease has been reported. There have been several studies dealing with complicated cardiac anomalies (5), vascular diseases $(6)$, respiratory diseases $(7,8)$, or anorectal malformations (9). However, there have so far been very few studies involving pediatric neoplastic diseases, in particular, regarding liver tumors $(10,11)$. A conventional CT examination and angiography were routinely performed for the preoperative evaluation for liver tumors in many institutions. Repeated CT scans were necessary to evaluate the efficacy of the preoperative chemotherapy. Therefore, serious problems were encountered regarding the accumulation of radiation exposure. On the other hand, general anesthesia is necessary for angiography and it is an invasive procedure for neonates and infants. MDCT more rapidly provides images than does conventional CT, which simplifies the process of sedation, and the MDCT provides a better quality of images and decreases the total accumulated dosage of radiation (12). MDCT also makes it possible to image the precise vascular anatomy including the anomalous branches, feeding arteries, or drainage veins (13).

As shown by the presented cases, the reconstructed images from MDCT were never inferior to those obtained by angiography. Anomalous vessel branches were detected in 3 of the 10 cases and 2 of them were confirmed by the findings by the subsequent angiography. Therefore, when a chemoembolization by TACE is not necessary, this MDCT reconstruction is considered to provide a sufficient evaluation of the vessels.

On the other hand, each image phase (arterial phase, equilibrium phase, portal phase) could be independently and simultaneously extracted or combined, respectively. These combined images can not be obtained by conventional CT procedures.

Furthermore, the software program for volumetry provides a proposed remnant liver volume and an optimal cut line of the liver. Various preoperative simulations can thus be considered. The residual liver volume was calculated to be above $40 \%$ for all the presented cases. This volumetric analysis therefore positively contributes to the safety of the procedure by assisting in the selection of the optimal operations.

Since the pediatric age ranges from the neonate to school children having an adult physical constitution, standard- 


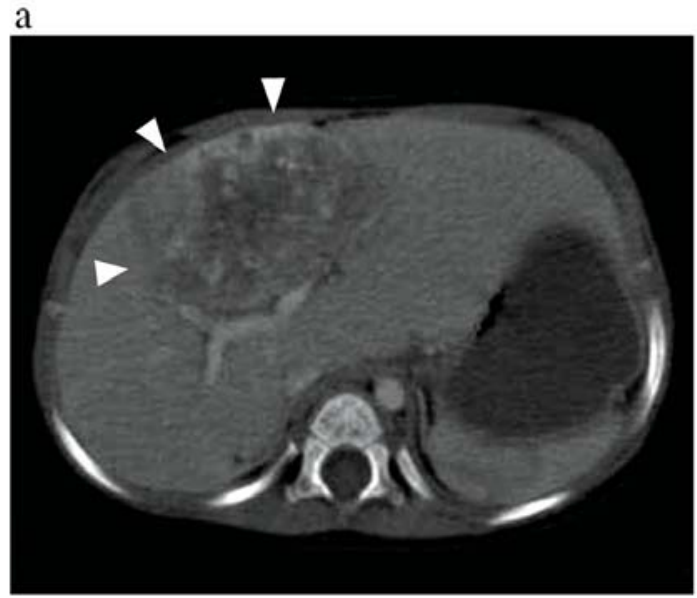

b

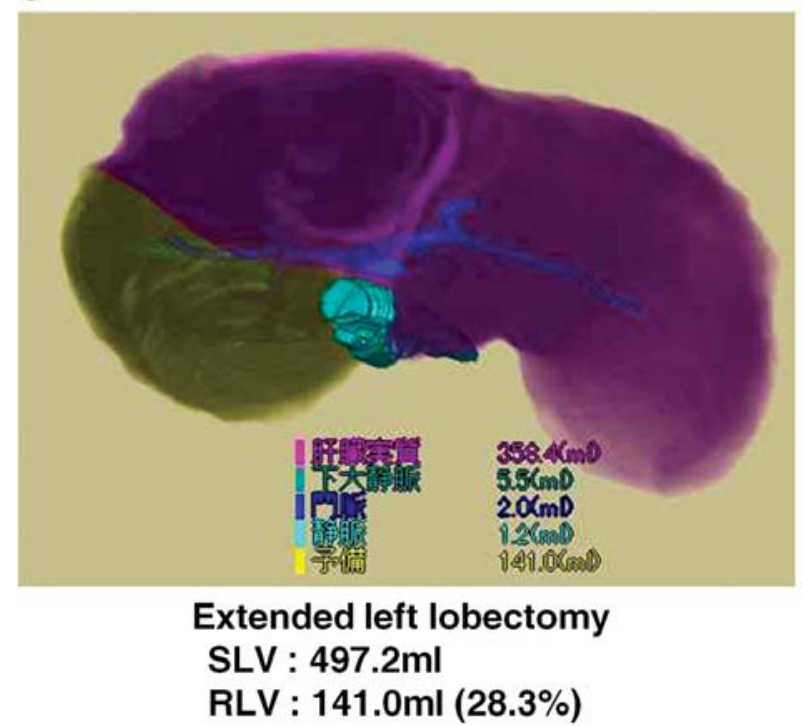

$\mathrm{c}$

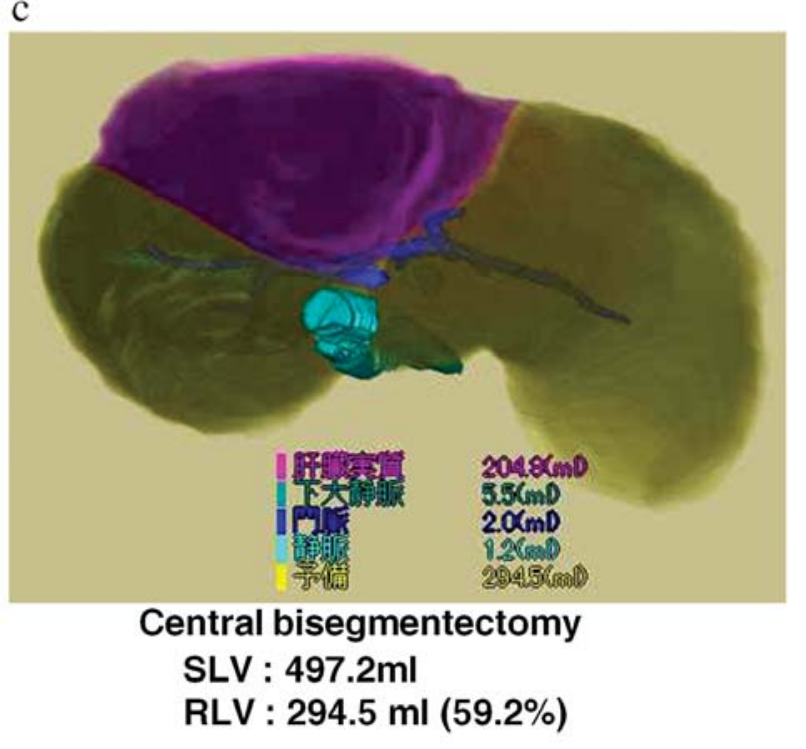

Figure 3. The volumetric analysis (case 2). (a) A 2-D image of a conventional CT scan. (b) The simulation of an extended left lobectomy. The standard liver volume (SLV) was $497.2 \mathrm{ml}$ and the residual liver volume (RLV) was $141.0 \mathrm{ml}(28.3 \%)$. (c) The simulation of a central bisegmentectomy. The residual liver volume was $294.5 \mathrm{ml}(59.2 \%)$.

ization of the regimen for each age group is considered to be necessary. Relatively large diameter vessels are necessary for the enhancing procedure, in spite of the difficulty of preserving the vascular route. The volume of the contrast medium, allergic events, and the timing of enhancement are still unsolved problems.

In conclusion, the reconstruction images by MDCT and the performance of a volumetric analysis provide extensive useful information for the preoperative evaluation. Since pediatric surgeons perform these reconstructive procedures themselves, it is possible to obtain the most adequate preoperative evaluation, in order to then select the optimal operation for each patient. The resolution of the specific problems in the pediatric area, and the development of more effective procedures is expected in the future.

\section{Acknowledgements}

We would like to thank Mr. Brian Quinn for reading the manuscript. This study was supported in part by a grant-in- aid for scientific research from the Japanese Society for Promotion of Science.

\section{References}

1. Yonemura Y, Taketomi A, Soejima Y, et al: Validity of preoperative volumetric analysis of congestion volume in living donor liver transplantation using three-dimensional computed tomography. Liver Transpl 11: 1556-1562, 2005.

2. Urata K, Kawasaki S, Matsunami H, et al: Calculation of child and adult standard liver volume for liver transplantation. Hepatology 21: 1317-1321, 1995.

3. Rubin GD, Dake MD, Napel SA, et al: Three-dimensional spiral CT angiography of the abdomen; initial crinical experience. Radiology 186: 147-152, 1993.

4. Heiken JP, Brink JA and Vannier MW: Spiral (herical) CT. Radiology 189: 647-656, 1993.

5. Westra SJ, Hill JA, Alejos JC, et al: Three-dimensional herical $\mathrm{CT}$ of pulmonary arteries in infants and children with congenital heart disease. Am J Radiol 173: 109-115, 1999.

6. Chan FP and Rubin GD: MDCT angiography of pediatric vascular diseases of the abdomen, pelvis, and extremities. Pediatr Radiol 35: 40-53, 2005.

7. Siegel MJ: Multiplanar and three-dimensional multi-detector row CT of thoracic vessels and airways in the pediatric population. Radiology 229: 641-650, 2003. 
8. Kosucu P, Ahmetoglu A, Koramaz I, et al: Low-dose MDCT and virtual bronchoscopy in pediatric patients with foreign body aspiration. Am J Roentgenol 184: 1706-1707, 2005.

9. Watanabe Y, Ikegami R, Takasu K, et al: Three-dimensional computed tomographic images of pelvic muscle in anorectal malformations. J Pediatr Surg 40: 1931-1934, 2005.

10. Fuchs J, Warmann SW, Szavay P, et al: Three dimensional visualization and virtual simulation of resection in pediatric solid tumors. J Pediatr Surg 40: 364-370, 2005.

11. Dong Q, Xu W, Jiang B, et al: Clinical application of computerized tomography 3-D reconstruction imaging for diagnosis and surgery in children with large liver tumors or tumors at the hepatic hilum. Pediatr Surg Int 23: 1045-1050, 2007.
12. Frush DP, Slack CC, Hollingsworth CL, et al: Computersimulated radiation dose reduction for abdominal multidetector CT of pediatric patients. Am J Roentgenol 179: 1107-1113, 2002.

14. Frericks BB, Caldarone FC, Nashan B, et al: 3D CT modeling of hepatic vessel architecture and volume calculation in living donated liver transplantation. Eur Radiol 14: 326-333, 2004. 